\title{
Anesthetic management of a patient with hypertrophic cardiomyopathy and patent ductus arteriosus
}

\author{
Hoon Jung, Haegyeong Lee, Jung-A Lim, Seong Wook Hong, and Dong Gun Lim \\ Department of Anesthesiology and Pain Medicine, School of Medicine, Kyungpook National University, Daegu, Korea
}

An anesthetic experience of hypertrophic cardiomyopathy (HCMP) and patent ductus arteriosus (PDA) simultaneously is rare. The authors report that a 30-year-old man who had HCMP with systolic anterior motion (SAM) and PDA simultaneously, successfully underwent an internal fixation of an orbital wall fracture under general anesthesia.

The 30-year-old, $157 \mathrm{~cm}, 43 \mathrm{~kg}$ man was admitted for internal fixation due to orbital wall fracture. The patient had a previous history of PDA at birth but didn't undergo PDA closure. He also had HCMP and was treated with beta-blockers.

The transthoracic echocardiography showed HCMP with an ejection fraction of 57\%, left ventricular outflow tract (LVOT) obstruction with SAM, left atrial enlargement, left ventricle hypertrophy (18-22 $\mathrm{mm}$ ), right ventricular hypertrophy, right ventricle infundibular stenosis, pulmonic valve calcification, and $6 \mathrm{~mm}$ PDA. The chest X-ray showed an increase in cardiothoracic ratio $66 \%$

Before anesthetic induction, a continuous hemodynamic monitoring device (FloTrac sensor, Edwards, Irvine, CA, USA) was inserted by cannulation of right radial artery. To induce anesthesia, propofol $2 \mathrm{mg} / \mathrm{kg}$ and rocuronium bromide $0.6 \mathrm{mg} /$ $\mathrm{kg}$ were intravenously administered slowly while cautiously observing blood pressure (BP) and endotracheal intubation was performed. Anesthesia was maintained with sevoflurane 1.5$2.5 \mathrm{vol} \%, \mathrm{O}_{2} 0.5 \mathrm{~L} / \mathrm{min}$, air $3.5 \mathrm{~L} / \mathrm{min}\left(\mathrm{FiO}_{2}=30 \%\right)$, and remifentanil $0.1 \mu \mathrm{g} / \mathrm{kg} / \mathrm{min}$. Mechanical ventilation was conducted with a tidal volume (TV) of $8 \mathrm{ml} / \mathrm{kg}$, respiratory rate (RR) of 10 breaths/min, a positive end expiratory pressure (PEEP) $5 \mathrm{~cm}$ $\mathrm{H}_{2} \mathrm{O}$, and mean airway pressure $15 \mathrm{cmH}_{2} \mathrm{O}$. Because $\mathrm{BP}$ started to show a gradual decrease after induction, $300 \mathrm{ml}$ Hartmann's solution and $0.8 \mu \mathrm{g} / \mathrm{kg} / \mathrm{min}$ phenylephrine were intravenously administered. The hemodynamic parameters remained stable, with BP 110/60-130/70 mmHg, heart rate (HR) 55-60 beats/ min, and stroke volume variation (SVV) $5-8 \%$ throughout the operation. And then, a transesophageal echocardiography (TEE) was inserted and showed no LVOT obstruction. The total surgery time was 20 minutes and the anesthetic time was 55 minutes. The patient was discharged on postoperative 3 day without any complications.

It is important to monitor $\mathrm{BP}$ and cardiac output (CO) by Flotrac before induction in order to predict and manage sudden and unexpected systemic hypotension (e.g., due to blood loss and hypovolemia) and triggering of dynamic LVOT obstruction.

$\mathrm{CO}$ and BP were continuously observed by Flotrac. Because of appropriate maintenance of preload and afterload by hemodynamic monitoring, TEE showed normal preload and contractility, and no LVOT obstruction with SAM after induction. The intraoperative cardiac index $\left(4.0-4.4 \mathrm{~L} / \mathrm{min} / \mathrm{m}^{2}\right)$ and stoke volume index $\left(64-81 \mathrm{ml} / \mathrm{beat} / \mathrm{m}^{2}\right)$ were within the normal range. We set the goal of maintaining SVV at $10 \%$ for the appropriate preload. It is also important to select anesthetic agents in HCMP patients that will depress myocardial function and lower systemic vascular resistance (SVR). Therefore, sevoflurane was revealed as the ideal inhalation anesthetic [1]. If the patient has diastolic dysfunction, abrupt increases in HR can decrease SV by shortening diastolic filling time. We used sevoflurane and remifentanil to minimize sympathetic stimulation.

For anesthetic management in PDA patients, the avoidance

Corresponding author: Hoon Jung, M.D., Department of Anesthesiology and Pain Medicine, School of Medicine, Kyungpook National University, 130, Dalgubeoldae-ro, Jung-gu, Daegu 700-705, Korea. Tel: 82-53-420-5863, Fax: 82-53-426-2760, E-mail: wing2392@naver.com (c) This is an open-access article distributed under the terms of the Creative Commons Attribution Non-Commercial License (http:// creativecommons.org/licenses/by-nc/3.0/), which permits unrestricted non-commercial use, distribution, and reproduction in any medium, provided the original work is properly cited. 
of increases in SVR and decreases in pulmonary vascular resistance (PVR) in order to prevent increases in left-to-right shunt is required [2]. Ventilatory management was used to maintain PEEP $5 \mathrm{cmH}_{2} \mathrm{O}$, end tidal $\mathrm{CO}_{2} 40 \mathrm{mmHg}$, and $\mathrm{FiO}_{2} 0.3$, in accordance with treatment of pulmonary hypertension.

Because high airway pressure in HCMP patients exaggerates LVOT obstruction [1], it is important to maintain adequate minute ventilation by reducing $\mathrm{TV}$ and increasing $\mathrm{RR}$ within the framework of PVR. This respiratory management is also applied to PDA patients. In the present report, mechanical ventilation was conducted with TV $350 \mathrm{ml}$, RR 10 breaths/min, mean airway pressure $15 \mathrm{cmH}_{2} \mathrm{O}$, and $\mathrm{FiO}_{2}$ 0.3.

Decreases in SVR should be avoided in HCMP, but increases in SVR in PDA. Direct measurement of SVR was required, however, central venous pressure (CVP) was not monitored because the surgery was minor and of short duration. Instead of CVP monitoring, the decreases in SVR was monitored by assessment of LVOT obstruction with TEE.
There are some reasons of hypotension in the HCMP patient. First, due to a decrease in SVR caused by anesthetic agents, and second, due to a decrease in preload caused by blood loss and NPO time. In this case, there were no bleeding event or dehydration symptoms. Therefore, phenylephrine was optimal drug to use in the HCMP patient to increase SVR and induce rebound bradycardia. Increasing SVR can affect left-to-right shunt in PDA, but the duration of this surgery was short, therefore this was not an issue. One hypotensive episode was experienced after induction of anesthesia in the patient, however it was possible to maintain CO and BP by continuous phenylephrine infusion according to our anesthetic plan.

In conclusion, anesthetic management in HCMP is required to increase SVR, and in PDA to decrease SVR in order to prevent aggravation of left-to-right shunt. The choice of anesthetic agents and appropriate hemodynamic monitoring are important in these contradictory anesthetic managements.

\section{References}

1. Poliac LC, Barron ME, Maron BJ. Hypertrophic cardiomyopathy. Anesthesiology 2006; 104: 183-92.

2. Franco SA, Hines RL. Congenital heart disease. In: Stoelting's anesthesia and co-existing disease. 6th ed. Edited by Hines RL, Marschall KE: Philadelphia, Elsevier Saunders. 2012, pp 48-72. 